\title{
Wissenschaftspreis 2013 - Ausschreibung
}

Die Deutsche Gesellschaft für Ultraschall in der Medizin vergibt Förderpreise für hervorragende wissenschaftliche Arbeiten auf dem Gebiet des medizinischen Ultraschalls. Die Förderpreise bestehen aus einer Urkunde und einem Geldbetrag.

Im Jahr 2013 werden 3 Preise ausgeschrieben: Der 1. Preis ist mit € 2500,- dotiert, der 2. mit €1500,-, der 3. mit €1000,-. Die Preise können geteilt werden, wenn mehrere gleichwertige Bewerbungen vorliegen.

Für den DEGUM-Wissenschaftspreis 2013 können Originalarbeiten in deutscher oder englischer Sprache berücksichtigt werden, die nach dem 31.12.2010 publiziert bzw. zur Publikation angenommen wurden. Übersichtsarbeiten finden keine Berücksichtigung.

Sind an der prämierten Arbeit mehrere Autoren beteiligt, erhält jede Person eine Urkunde, der Geldbetrag wird entsprechend geteilt.

Da der Preis der Förderung des Nachwuchses dienen soll, sind Habilitationsschriften sowie Arbeiten von leitenden Ärzten von der Einreichung als Erstautor ausgenommen.

Jeder Bewerber (auch Mitautor) kann nur eine Arbeit einreichen.

Der Wissenschaftsbeirat der DEGUM bewertet die Arbeit auf der Grundlage von 2
Gutachten und entscheidet über die Preisvergabe, seine Entscheidung ist endgültig und nicht anfechtbar.

Die Bewerbung ist in elektronischer Form bis zum 19.04.2013 an die DEGUM-Geschäftsstelle zu richten. Der Bewerbung ist - ebenfalls in elektronischer Form - ein Sonderdruck oder ein entsprechend vollständiges Manuskriptdokument mit einer die Annahme zur Publikation bestätigenden Stellungnahme des Herausgebers der Zeitschrift beizufügen.

München im November 2012

Dr. S. Nöldeke, Präsident der DEGUM Prof. Dr. M. Görtler, Sekretär der DEGUM 